\title{
A mean field equation on a torus: one-dimensional symmetry of solutions
}

\author{
XAVIER CABRÉ \\ Institució Catalana de Recerca i Estudis Avançats (ICREA) and \\ Universitat Politècnica de Catalunya, Dep. de Matemàtica Aplicada I, \\ Diagonal 647, 08028 Barcelona, Spain, xavier.cabre@upc.es \\ Marcello LuCia \\ Rutgers University, Dep. of Mathematics, Hill-Center, \\ PISCATAWAY, NJ 08854, USA, mlucia@math.rutgers.edu \\ MANEl SANChÓN \\ Universitat Politècnica de Catalunya, Dep. de Matemàtica Aplicada I, \\ Diagonal 647, 08028 Barcelona, Spain, Manuel.Sanchon@upc.es
}

\begin{abstract}
We study the equation

$$
-\Delta u=\lambda\left(\frac{e^{u}}{\int_{\Omega_{\varepsilon}} e^{u}}-\frac{1}{\left|\Omega_{\varepsilon}\right|}\right) \quad \text { in } \Omega_{\varepsilon}
$$

for $u \in E$, where $E=\left\{u \in H^{1}\left(\Omega_{\varepsilon}\right): u\right.$ is doubly periodic, $\left.\int_{\Omega_{\varepsilon}} u=0\right\}$ and $\Omega_{\varepsilon}$ is a rectangle of $\mathbb{R}^{2}$ with side lengths $1 / \varepsilon$ and $1,0<\varepsilon \leq 1$. We establish that every solution depends only on the $x$-variable when $\lambda \leq \lambda^{*}(\varepsilon)$, where $\lambda^{*}(\varepsilon)$ is an explicit positive constant depending on the maximum conformal radius of the rectangle. As a consequence, we obtain an explicit range of parameters $\varepsilon$ and $\lambda$ in which every solution is identically zero. This range is optimal for $\varepsilon \leq 1 / 2$.
\end{abstract}




\section{Introduction}

In this article we study solutions of the equation

$$
-\Delta u=\lambda\left(\frac{e^{u}}{\int_{\Omega_{\varepsilon}} e^{u}}-\frac{1}{\left|\Omega_{\varepsilon}\right|}\right) \quad \text { in } \Omega_{\varepsilon}, \quad u \in E,
$$

where

$$
\Omega_{\varepsilon}=\left(-\frac{1}{2 \varepsilon}, \frac{1}{2 \varepsilon}\right) \times\left(-\frac{1}{2}, \frac{1}{2}\right) \subset \mathbb{R}^{2}, \quad 0<\varepsilon \leq 1,
$$

and

$$
E:=\left\{u: \Omega_{\varepsilon} \rightarrow \mathbb{R}: u \in H^{1}\left(\Omega_{\varepsilon}\right), u \text { doubly periodic, } \int_{\Omega_{\varepsilon}} u=0\right\} .
$$

Solutions of problem (1) are critical points of the functional $\mathcal{J}^{\lambda, \varepsilon}: E \rightarrow \mathbb{R}$ defined by

$$
\mathcal{J}^{\lambda, \varepsilon}(u)=\frac{1}{2} \int_{\Omega_{\varepsilon}}|\nabla u|^{2}-\lambda \log \left(\frac{1}{\left|\Omega_{\varepsilon}\right|} \int_{\Omega_{\varepsilon}} e^{u}\right) .
$$

Note that equation (1) and the functional $\mathcal{J}^{\lambda, \varepsilon}$ are both invariant under dilations. Thus, there is no loss of generality in taking the vertical size of the rectangle $\Omega_{\varepsilon}$ to be 1 .

In this paper we find an explicit range of parameters $\varepsilon$ and $\lambda$ in which every solution of (1) is necessarily one-dimensional (1D solution for short), in the sense that it depends only on the $x$-variable. As a consequence, we obtain a second range of parameters in which every solution is zero. This range is optimal for $\varepsilon \leq 1 / 2$.

Equations of the type (1) arise in Onsager's vortex theory for 1 specie in a torus or in $S^{2}$. Another motivation comes from $(2+1)$-dimensional Chern-Simons gauge theory (see [HKP90], [JW90], [D96], [T96], [DJLW98], [NT99]). More precisely, when the vortex number $N=1$ and the ChernSimons coupling constant tends to zero, Tarantello [T96] showed that the asymptotic behavior of some solutions solve the limiting equation

$$
-\Delta u=4 \pi\left(\frac{K(x) e^{u}}{\int_{\Omega_{\varepsilon}} K(x) e^{u}}-\frac{1}{\left|\Omega_{\varepsilon}\right|}\right),
$$

where $K$ is a preassigned nonnegative function. Here we treat the case $K \equiv 1$.

When $\lambda \leq 0$, it is easy to verify that the functional $\mathcal{J}^{\lambda, \varepsilon}$ is strictly convex. As a consequence, zero is the only solution of (1) when $\lambda \leq 0$.

Note also that, by Jensen's inequality, $\left(1 /\left|\Omega_{\varepsilon}\right|\right) \int_{\Omega_{\varepsilon}} e^{u} \geq 1$ for every $u \in E$. As a consequence, the functional $\mathcal{J}^{\lambda, \varepsilon}$ is nonincreasing in $\lambda$. 
By Moser-Trudinger inequality [M71], it is easy to verify that the functional $\mathcal{J}^{\lambda, \varepsilon}$ is bounded from below if and only if $\lambda \leq 8 \pi$. In addition, one can check that for $\lambda<8 \pi, \mathcal{J}^{\lambda, \varepsilon}$ is coercive and lower semivontinuous, and hence it admits an absolute minimizer (minimizer from now on). Note that since zero is a solution of (1), zero could be a minimizer of $\mathcal{J}^{\lambda, \varepsilon}$. Note also that $\mathcal{J}^{\lambda, \varepsilon}(0)=0$. Similar existence results when $\lambda<8 \pi$ have been proved in [CLMP92] and [Ki93] for the functional $\mathcal{J}^{\lambda, \varepsilon}$ considered in $H_{0}^{1}$ (Dirichlet boundary conditions).

For $\lambda=8 \pi$, the existence of a minimizer $u \in E$ of $\mathcal{J}^{\lambda, \varepsilon}$ is a more subtle question. Nolasco and Tarantello [NT99] established that a minimizer exists for every rectangle.

In the case where the functional is unbounded from below, $\lambda>8 \pi$, Struwe and Tarantello [ST98] proved that the trivial solution $u \equiv 0$ remains a strict local minimum for $\lambda<4 \pi^{2}$ and $\varepsilon=1$, and that the functional $\mathcal{J}^{\lambda, 1}$ exhibits a mountain-pass structure leading to the existence of truly two-dimensional solutions for each $\lambda \in\left(8 \pi, 4 \pi^{2}\right)$.

On the other hand, Ricciardi and Tarantello [RT98] proved that $\lambda>4 \pi^{2} \varepsilon$ is a necessary and sufficient condition for the existence of at least one nonzero one-dimensional solution. Moreover, when $4 \pi^{2} \varepsilon<8 \pi$ (i.e., $\varepsilon<2 / \pi$; see Figure 1 below) and $\lambda \in\left(4 \pi^{2} \varepsilon, 8 \pi\right]$, an appropriate choice of a test function shows that any minimizer is nonzero (see [RT98] or Proposition 4.1 below).

In this article we consider the parameter range $\lambda \leq 8 \pi$. We find an explicit positive function $\lambda^{*}(\varepsilon)$ of $\varepsilon$ such that: if $\lambda \leq \lambda^{*}(\varepsilon)$ then every solution is 1D. The function $\lambda^{*}(\varepsilon)$ depends on the maximum conformal radius $R_{\varepsilon}$ of $\Omega_{\varepsilon}$ (see Appendix A for the definition and properties of $R_{\varepsilon}$ ). The explicit expression of $\lambda^{*}(\varepsilon)$ in terms of $R_{\varepsilon}$ is given in the following theorem.

Theorem 1.1. Let $R_{\varepsilon}$ be the maximum conformal radius of the rectangle $\Omega_{\varepsilon}$ and let $u$ be a solution of (1). If

$$
\lambda \leq \lambda^{*}(\varepsilon):=\frac{16 \pi^{3}}{\pi^{2}+2 / R_{\varepsilon}^{2}+\sqrt{\left(\pi^{2}+2 / R_{\varepsilon}^{2}\right)^{2}-8 \pi^{3} /\left|\Omega_{\varepsilon}\right|}}
$$

then $\partial_{y} u \equiv 0$. That $i s, u$ is a $1 D$ solution, meaning that $u$ depends only on the $x$-variable.

The function $\lambda^{*}$ satisfies $0<\lambda^{*}(\varepsilon)<8 \pi$ for all $\varepsilon \in(0,1]$. It is pictured with high precision in Figure 1 below using expression (4) and the explicit formula (19) (that we prove in an appendix) for the maximum conformal radius $R_{\varepsilon}$ of the rectangle. Note also that $\lim _{\varepsilon \downarrow 0} \lambda^{*}(\varepsilon)=(2 / 3) 8 \pi$.

Since, by [RT98], every 1D solution of (1) is zero when $\lambda \leq 4 \pi^{2} \varepsilon$, our theorem implies that every solution is identically zero for $\lambda \leq \min \left\{\lambda^{*}(\varepsilon), 4 \pi^{2} \varepsilon\right\}$ 


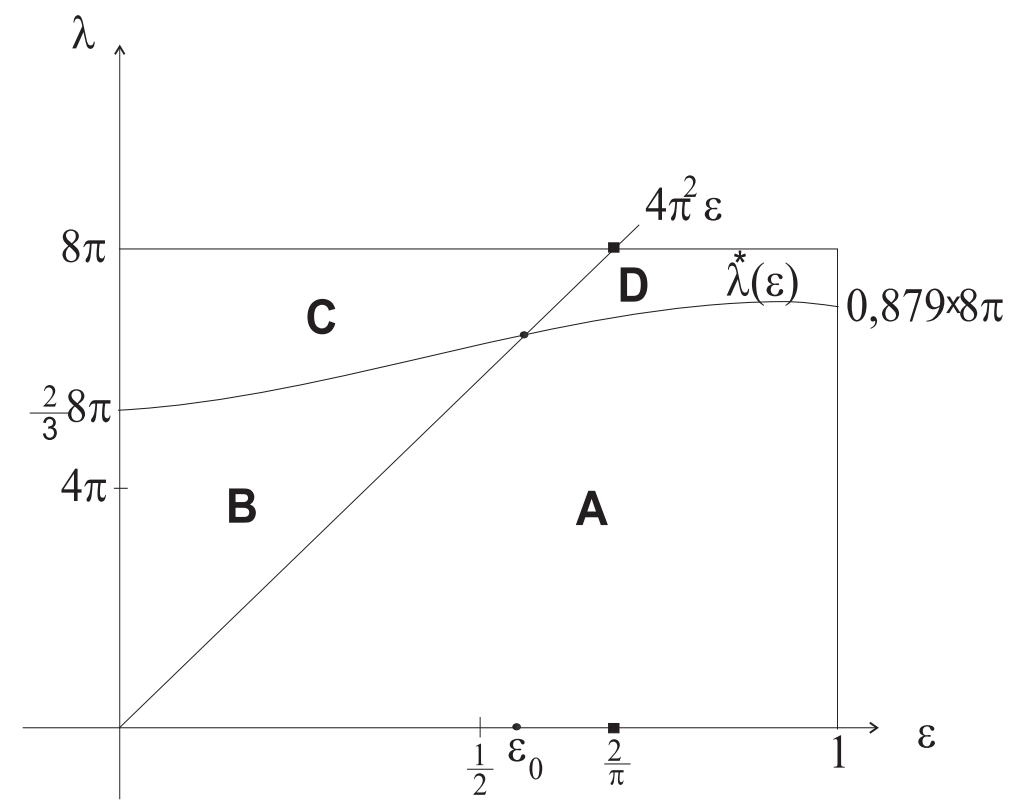

Figure 1. In region $A$, every solution is zero. In region $B$, every solution is $1 D$; moreover, any minimizer is nonzero. In region $C$, there exists a nonzero minimizer. In region $D$, the existence of a nonzero solution is an open problem.

(that is, in region $\mathrm{A}$ of the figure). As a consequence, let $\varepsilon_{0}$ be the unique solution of $\lambda^{*}\left(\varepsilon_{0}\right)=4 \pi^{2} \varepsilon_{0}$ and let $\varepsilon \leq \varepsilon_{0} \simeq 0.509$. Since the infimum of $\mathcal{J}^{\lambda, \varepsilon}$ is strictly negative for $\lambda>4 \pi^{2} \varepsilon$ (see [RT98] or Proposition 4.1 below), then (1) admits a nonzero solution if and only if $\mathcal{J}^{\lambda, \varepsilon}$ admits a nonzero minimizer, and if and only if $\lambda \in\left(4 \pi^{2} \varepsilon, 8 \pi\right]$. That is, we have the following result.

Corollary 1.2. If $\lambda \leq \min \left\{\lambda^{*}(\varepsilon), 4 \pi^{2} \varepsilon\right\}$, where $\lambda^{*}$ is given in Theorem 1.1, then $u \equiv 0$ is the unique solution of (1).

Moreover, let $\varepsilon_{0}$ be the unique solution of $\lambda^{*}\left(\varepsilon_{0}\right)=4 \pi^{2} \varepsilon_{0}$. Assume that $\varepsilon \leq \varepsilon_{0}$ and $0<\lambda \leq 8 \pi$. Then, (1) admits a nonzero solution if and only if $\mathcal{J}^{\lambda, \varepsilon}$ admits a nonzero minimizer, and if and only if $\lambda \in\left(4 \pi^{2} \varepsilon, 8 \pi\right]$.

In case $8 \pi \leq 4 \pi^{2} \varepsilon$ (i.e., $\varepsilon \geq 2 / \pi$ ), the existence or nonexistence of nonzero minimizers, and also of nonzero solutions, for some $\lambda \leq 8 \pi$ were open questions. Since $\lambda^{*}(1) \simeq 8 \pi \times 0.879$, from Theorem 1.1 we deduce that for the square $(\varepsilon=1)$ and $\lambda=4 \pi$, problem (1) admits only the zero solution. This improves a result of [ST98] stating that (1) admits only the zero solution for $\lambda$ small enough. Note that $\lambda=4 \pi$ is a relevant parameter since it corresponds to one vortex, as in equation (3). 
In region $\mathrm{D}$ of Figure 1 it is still not known if there exists a nonzero solution.

The main tool in the proof of Theorem 1.1 is estimate (11) of Proposition 2.2 below. This estimate is an $L^{\infty}$ bound for solutions of (1) for every $\lambda<8 \pi$. This kind of estimates for problem (1) are delicate to establish (see Remark 2.3 for more comments on this direction). Our estimate (11) is not the best possible, but it is completely explicit in terms of $\lambda$ and $\varepsilon$. The techniques that lead to our $L^{\infty}$ bound are conformal transformations and an isoperimetric inequality due to Bandle [B73], stated in Proposition 2.1 below.

Our approach is very close to a work of Kiessling [Ki95] that established uniqueness results for similar equations, with the difference that here we take into account the maximum conformal radius of the domain $\Omega_{\varepsilon}$.

The article is organized as follows. In Section 2 we establish the $L^{\infty}$ bound (11) for solutions of problem (1). In Section 3 we prove a Poincaré inequality, Theorem 1.1, and Corollary 1.2. In Section 4 we establish an upper bound for inf $\mathcal{J}^{\lambda, \varepsilon}$ and state some symmetry properties of the minimizers of $\mathcal{J}^{\lambda, \varepsilon}$. Finally, in Appendix A we define the maximum conformal radius of a domain and we prove an explicit formula for it when the domain is a rectangle.

\section{An $L^{\infty}$ bound for solutions}

This section is concerned with some isoperimetric type inequalities and supremum bounds for subsolutions of the local equation $-\Delta v=e^{v}$. We prove estimate (11) of Proposition 2.2, which is the bound that we will use in the following section to establish Theorem 1.1.

Proposition 2.1. Let $\Omega$ be a simply connected bounded domain of $\mathbb{R}^{2}$ and $v \in C^{2}(\bar{\Omega})$ be a function satisfying

$$
-\Delta v \leq e^{v} \quad \text { in } \Omega
$$

Then, the following hold:

(i) (Bol's inequality) Given $\omega \subset \subset \Omega$, with $\omega$ smooth and simply connected, we have

$$
L(\partial \omega)^{2} \geq \frac{1}{2}\{8 \pi-m(\omega)\} m(\omega)
$$

where

$$
L(\partial \omega)=\int_{\partial \omega} e^{v / 2} d l \quad \text { and } \quad m(\omega)=\int_{\omega} e^{v} d x d y
$$


(ii) (Bandle) Let $\Omega=B_{R}=B_{R}(0)$ be a ball of radius $R$ and assume that $m:=\int_{B_{R}} e^{v}<8 \pi$. Then,

$$
e^{v(0)} \leq \frac{8 \pi}{\left|B_{R}\right|} \frac{m}{8 \pi-m} .
$$

(iii) Let $\Omega=\Omega_{\varepsilon}=(-1 /(2 \varepsilon), 1 /(2 \varepsilon)) \times(-1 / 2,1 / 2)$. Assume that $m:=$ $\int_{\Omega_{\varepsilon}} e^{v}<8 \pi$ and let $R_{\varepsilon}$ be the maximum conformal radius of $\Omega_{\varepsilon}$. Then,

$$
e^{v(0)} \leq \frac{8}{R_{\varepsilon}^{2}} \frac{m}{8 \pi-m}
$$

Statements (i) and (ii) are well known. The novelty here is statement (iii) in rectangles, which plays a crucial role in our main result. Note that, since $v$ is periodic, the maximum of $v$ can be located at the origin after a translation. As a consequence of this, Proposition 2.1(iii) gives a bound for $\exp \left\{\max _{\Omega_{\varepsilon}} v\right\}$.

Proof of Proposition 2.1.

(i) For the sake of completeness we include here a sketch of the proof. We refer to [B80] (Section I.3.6), [S92] or [S94] for detailed proofs.

Consider the conformal metric $d \sigma^{2}=e^{v} d x d y$ in $\omega$. Let $\varphi=d_{\sigma}(\cdot, \partial \omega)$ be the distance to $\partial \omega$ induced by this metric. For $t \geq 0$, consider $\Gamma(t)=$ $\{(x, y) \in \omega: \varphi(x, y)=t\}$ and $\omega(t)=\{(x, y) \in \omega: \varphi(x, y)>t\}$. The goal is to find a relation between

$$
L(t):=\int_{\Gamma(t)} d \sigma \quad \text { and } \quad m(t):=\int_{\omega(t)} d \sigma^{2} .
$$

Note that for $t=0$ these quantities coincide with $L(\partial \omega)$ and $m(\omega)$, respectively, as defined in (6).

By the coarea formula, we have

$$
m(t)=\int_{\omega(t)} e^{v} d x d y=\int_{t}^{t^{*}} d s \int_{\Gamma(s)} \frac{e^{v}}{|\nabla \varphi|} d l
$$

where $d l$ is the Euclidean length element and $t^{*}=\sup _{\omega} \varphi$. Moreover, $m$ is differentiable at almost every $t$ and, since $|\nabla \varphi|=e^{v / 2}$ (where $\nabla$ denotes always the Euclidean gradient), we have that

$$
m^{\prime}(t)=-\int_{\Gamma(t)} \frac{e^{v}}{|\nabla \varphi|} d l=-\int_{\Gamma(t)} e^{v / 2} d l=-\int_{\Gamma(t)} d \sigma=-L(t)
$$

for almost every $t$. 
Hartman [H64] showed that $\Gamma(t)$ is a piecewise $C^{2}$ curve for a.e. $t$. Denote the Euclidean curvature of $\Gamma(t)$ by $K_{\Gamma(t)}$, and note that $d / d t=e^{-v / 2} \partial / \partial \nu$, where $\nu$ is the Euclidean exterior unit normal to $\Gamma(t)$ and $d / d t$ denotes derivative of a function with respect to $t$ in the orthogonal direction to $\Gamma(t)$. In general, the function $L$ is not everywhere continuous, but for a.e. $t$, $L(t)=\int_{\Gamma(t)} e^{v / 2} d l$ is differentiable at $t$. At such points, by the Gauss-Bonnet formula in case that $\Gamma(t)$ has no angles, we obtain

$$
\begin{aligned}
L^{\prime}(t) & =-\int_{\Gamma(t)} K_{\Gamma(t)} d l-\int_{\Gamma(t)} e^{-v / 2} \frac{\partial e^{v / 2}}{\partial \nu} d l=-2 \pi-\frac{1}{2} \int_{\Gamma(t)} \frac{\partial v}{\partial \nu} d l \\
& =-2 \pi-\frac{1}{2} \int_{\omega(t)} \Delta v d x d y \leq-2 \pi+\frac{1}{2} m(t) .
\end{aligned}
$$

It can be shown that this inequality, $L^{\prime}(t) \leq-2 \pi+m(t) / 2$, remains valid when $\Gamma(t)$ has angles, and hence for a.e. $t$.

Multiply now this differential inequality by $2 L(t)$ and integrate over $\left[0, t^{*}\right]$, to formally obtain

$$
L(\partial \omega)^{2} \geq 4 \pi m(\omega)-\frac{1}{2} m(\omega)^{2}=\frac{1}{2}(8 \pi-m(\omega)) m(\omega),
$$

where we have used that $L\left(t^{*}\right) \geq 0$ and $m\left(t^{*}\right)=0$. In [H64] it is shown that this formal integration is allowed.

(ii) This part is a result due to Bandle ([B73], [B80]). Its proof uses Bol's inequality and, for the sake of completeness, we include it here following [B80] (Lemma 1.2, page 43). Set

$$
B_{R}=B_{R}(0), \quad B_{r}=B_{r}(0), \quad \text { and } \quad K(r):=\int_{B_{r}} e^{v},
$$

for $0<r<R$.

Using Schwarz and Bol's inequalities, we have

$$
K^{\prime}(r)=\int_{\partial B_{r}} e^{v} \geq \frac{1}{2 \pi r}\left(\int_{\partial B_{r}} e^{v / 2}\right)^{2} \geq \frac{1}{4 \pi r}\{8 \pi-K(r)\} K(r) .
$$

Since $8 \pi-K(r)>0$ by hypothesis, we obtain

$$
\frac{K^{\prime}(r)}{\{8 \pi-K(r)\} K(r)} \geq \frac{1}{4 \pi r} .
$$

Integrating from $r_{0}$ to $R$, and letting $r_{0} \rightarrow 0$, it is easy to conclude

$$
e^{v(0)} \leq \frac{8 K(R)}{R^{2}\{8 \pi-K(R)\}}=\frac{8 \pi}{\left|B_{R}\right|} \frac{m}{8 \pi-m} .
$$


(iii) This result follows from the observation that the inequality of part (ii) is conformally invariant. More precisely, let $R_{\varepsilon}$ be the maximum conformal radius of $\Omega_{\varepsilon}$. We know (see Appendix A) that there is a conformal map $\varphi: B_{1}(0) \rightarrow \Omega_{\varepsilon}$, one to one and onto, such that $\varphi(0)=0$ and $\varphi^{\prime}(0)=R_{\varepsilon}>0$. Given $v \in C^{2}\left(\overline{\Omega_{\varepsilon}}\right)$, it is easy to check that

$$
\Delta(v \circ \varphi)=\left|\varphi^{\prime}\right|^{2} \Delta v \circ \varphi \quad \text { and } \quad \Delta\left(\log \left|\varphi^{\prime}\right|^{2}\right)=0 .
$$

Thus, by setting $\tilde{v}=v \circ \varphi+\log \left|\varphi^{\prime}\right|^{2}$, we have that

$$
-\Delta v \leq e^{v} \text { in } \Omega_{\varepsilon} \quad \text { if and only if }-\Delta \tilde{v} \leq e^{\tilde{v}} \text { in } B_{1} .
$$

Note that $\int_{B_{1}} e^{\tilde{v}}=\int_{\Omega_{\varepsilon}} e^{v}$ and $e^{v(0)}=e^{\tilde{v}(0)} / R_{\varepsilon}^{2}$. Hence, from part (ii) applied to $\tilde{v}$ with $R=1$, we immediately obtain (8).

Next, we use Proposition 2.1(iii) to obtain the following estimate for solutions of our nonlocal equation (1).

Proposition 2.2. Let $0<\lambda<8 \pi$, $u$ be a solution of (1), and $R_{\varepsilon}$ be the maximum conformal radius of $\Omega_{\varepsilon}$. Then,

$$
\frac{e^{u}}{\int_{\Omega_{\varepsilon}} e^{u}} \leq\left(\frac{8}{R_{\varepsilon}^{2}}-\frac{\lambda}{\left|\Omega_{\varepsilon}\right|}\right) \frac{1}{8 \pi-\lambda} \quad \text { in } \Omega_{\varepsilon} .
$$

Proof. Setting

$$
v:=u+\log \lambda-\log \int_{\Omega_{\varepsilon}} e^{u}
$$

we have

$$
-\Delta v=-\Delta u=\lambda\left(\frac{e^{u}}{\int_{\Omega_{\varepsilon}} e^{u}}-\frac{1}{\left|\Omega_{\varepsilon}\right|}\right) \leq \lambda \frac{e^{u}}{\int_{\Omega_{\varepsilon}} e^{u}}=e^{v}
$$

and

$$
\int_{\Omega_{\varepsilon}} e^{v}=\lambda
$$

Since $u$ is doubly periodic, we may place its maximum at the origin. Hence, from Proposition 2.1(iii) we obtain

$$
e^{v} / \lambda \leq B_{0}:=\frac{8}{R_{\varepsilon}^{2}}(8 \pi-\lambda)^{-1} .
$$

Estimate (12) holds for every solution $u$. In particular it holds for $u \equiv 0$, in which case $v \equiv \log (\lambda \varepsilon)$. Using this $v$ in (12) and letting $\lambda \downarrow 0$, we deduce

$$
\varepsilon \leq \frac{1}{\pi R_{\varepsilon}^{2}}
$$


an inequality related to the maximum conformal radius that we will use in several future occasions. First, (13) implies that the constant $B_{0}$ defined by (12) satisfies $B_{0}>\varepsilon$, whichever $0<\lambda<8 \pi$ is.

Assume now the existence of a constant $B_{n}>\varepsilon$ such that $e^{v} / \lambda \leq B_{n}$. Then

$$
-\Delta v=\left(1-\frac{\lambda}{\left|\Omega_{\varepsilon}\right| e^{v}}\right) e^{v} \leq\left(1-\frac{1}{\left|\Omega_{\varepsilon}\right| B_{n}}\right) e^{v} .
$$

Since $B_{n}>\varepsilon$, we have $1-1 /\left(\left|\Omega_{\varepsilon}\right| B_{n}\right)>0$ and hence the function $w:=$ $v+\log \left\{1-1 /\left(\left|\Omega_{\varepsilon}\right| B_{n}\right)\right\}$ is a new subsolution of $-\Delta w=e^{w}$. Moreover, it satisfies

$$
\int_{\Omega_{\varepsilon}} e^{w}=\left(1-\frac{1}{\left|\Omega_{\varepsilon}\right| B_{n}}\right) \lambda<\lambda<8 \pi
$$

Applying Proposition 2.1(iii) to $w$ we deduce that

$$
e^{v} / \lambda \leq B_{n+1}:=\frac{8}{R_{\varepsilon}^{2}}\left(8 \pi-\lambda+\frac{\lambda}{\left|\Omega_{\varepsilon}\right| B_{n}}\right)^{-1}
$$

Note that $B_{n+1}>\varepsilon$, since $-\lambda+\lambda /\left(\left|\Omega_{\varepsilon}\right| B_{n}\right)<0$.

Hence, we have obtained a sequence $\left(B_{n}\right)$ defined by

$$
B_{0}=\frac{8}{R_{\varepsilon}^{2}}(8 \pi-\lambda)^{-1}, \quad B_{n+1}=\frac{8}{R_{\varepsilon}^{2}}\left(8 \pi-\lambda+\frac{\lambda}{\left|\Omega_{\varepsilon}\right| B_{n}}\right)^{-1},
$$

with $B_{n}>\varepsilon$ and such that $e^{v} / \lambda \leq B_{n}$ for all $n$. Note that $B_{1}<B_{0}$ follows immediately from the definition of these two constants. Now, by induction, the recurrence relation leads to $B_{n+1}<B_{n}$. Hence, the sequence is decreasing, and we can use the recurrence relation to find

$$
\lim _{n \rightarrow+\infty} B_{n}=\left(\frac{8}{R_{\varepsilon}^{2}}-\frac{\lambda}{\left|\Omega_{\varepsilon}\right|}\right)(8 \pi-\lambda)^{-1} .
$$

We conclude that

$$
\frac{e^{u}}{\int_{\Omega_{\varepsilon}} e^{u}}=e^{v} / \lambda \leq\left(\frac{8}{R_{\varepsilon}^{2}}-\frac{\lambda}{\left|\Omega_{\varepsilon}\right|}\right)(8 \pi-\lambda)^{-1},
$$

which is estimate (11).

Remark 2.3. In [DJLW97] and [NT98] it is proved that for minimizers $u$ and $\lambda \leq 8 \pi$, the left hand side of (11) is uniformly bounded by a constant $C(\varepsilon)$ depending only on $\varepsilon$. Actually, from a result of Chen and Lin [CL], such a uniform a priori estimate up to $\lambda \leq 8 \pi$ also holds for every solution $u$. However, these proofs use blow-up arguments and hence do not give an 
explicit value for such a constant $C(\varepsilon)$. This is the reason why these estimates up to $\lambda \leq 8 \pi$ cannot be used in our future arguments to obtain explicit ranges of parameters as in Theorem 1.1 and Corollary 1.2. Instead, Proposition 2.2 gives an explicit estimate. However, it is not optimal for $\varepsilon$ fixed since it blows-up as $\lambda \uparrow 8 \pi$.

\section{A Poincaré estimate. Proof of Theorem 1.1 and Corollary 1.2}

To prove our results, we use the apriori estimate (11) of last section together with the following Poincaré estimate for the $y$-partial derivative of every solution.

Proposition 3.1. Let $u$ be a solution of (1) with its maximum located at the origin, and let $\psi:=\partial_{y} u$. Then,

$$
\int_{-1 / 2}^{1 / 2} \psi(x, y) d y=0 \quad \text { for all } x \in[-1 /(2 \varepsilon), 1 /(2 \varepsilon)]
$$

and, if $\psi \not \equiv 0$, we have

$$
4 \pi^{2} \int_{\Omega_{\varepsilon}} \psi^{2}<\int_{\Omega_{\varepsilon}}|\nabla \psi|^{2}
$$

Proof. Note that (14) is an immediate consequence of the fact that $u$ is periodic. To prove inequality (15), let us define

$$
V:=\left\{\phi \in H^{1}\left(\Omega_{\varepsilon}\right): \phi \text { is doubly periodic and } \int_{-1 / 2}^{1 / 2} \phi(x, y) d y=0 \quad \forall x\right\} .
$$

Note that $\psi=\partial_{y} u \in V$. Using Fourier series, it is easy to check that

$$
\mu:=\inf _{\phi \in V \backslash\{0\}} \frac{\int_{\Omega_{\varepsilon}}|\nabla \phi|^{2}}{\int_{\Omega_{\varepsilon}} \phi^{2}}
$$

is achieved by some $w \in V \backslash\{0\}$ satisfying

$$
\begin{gathered}
-\Delta w=\mu w, \quad w(x, y)=f(x) g(y) \\
-f^{\prime \prime}(x)=\alpha f(x), \quad \text { and } \quad-g^{\prime \prime}(y)=\beta g(y), \quad \int_{-1 / 2}^{1 / 2} g(y) d y=0 .
\end{gathered}
$$


Moreover, $\mu=\alpha+\beta$.

The periodicity for $g$ and the fact that it has mean zero imply that

$$
\beta>0, \quad \beta=(2 n \pi)^{2} \quad \text { and } \quad g(y)=A \sin (2 n \pi y) .
$$

The periodicity for $f$ imply that $\alpha \geq 0$. Hence, the least eigenvalue is $\alpha=0$, corresponding to $f$ identically constant. Therefore, the first eigenvalue in $V$ is given by $\mu=4 \pi^{2}$.

Therefore, recalling that $\psi:=\partial_{y} u \in V$, we get from the above discussion that

$$
4 \pi^{2} \int_{\Omega_{\varepsilon}} \psi^{2} \leq \int_{\Omega_{\varepsilon}}|\nabla \psi|^{2}
$$

Finally, assume that $\psi \not \equiv 0$ and, arguing by contradiction, that equality in (16) holds. Then, $\psi=\partial_{y} u$ is a multiple of the function $\sin (2 \pi y)$ and not identically zero. Therefore, there exists some function $\widetilde{u} \in E$ depending only in the variable $x$ such that

$$
u(x, y)=\widetilde{u}(x)+t \cos (2 \pi y)
$$

with $t \neq 0$ ( since $\left.\partial_{y} u \not \equiv 0\right)$. Since $u$ is a solution to problem (1) we obtain

$$
-\widetilde{u}^{\prime \prime}(x)+4 \pi^{2} t \cos (2 \pi y)=\lambda\left(\frac{e^{\widetilde{u}(x)} e^{t \cos (2 \pi y)}}{\int_{\Omega_{\varepsilon}} e^{\widetilde{u}(x)} e^{t \cos (2 \pi y)}}-\frac{1}{\left|\Omega_{\varepsilon}\right|}\right) .
$$

Taking $x=0$ in the last equality and using that $\left\{1, \cos (2 \pi y), e^{t \cos (2 \pi y)}\right\}$ are linearly independent functions in $[-1 / 2,1 / 2]$, we obtain $t=0$, a contradiction.

Proof of Theorem 1.1. Let $u$ be a solution of (1). We may assume that $\lambda>0$ since, as mentioned in the Introduction, when $\lambda \leq 0$ the functional $\mathcal{J}^{\lambda, \varepsilon}$ is strictly convex and hence zero is the only solution of (1).

After a translation we may assume that the maximum of $u$ is located at the origin. Set $\psi:=\partial_{y} u$ and assume that $\psi \not \equiv 0$. Differentiating equation (1) with respect to $y$, we obtain

$$
-\Delta \psi=\lambda \frac{e^{u}}{\int_{\Omega_{\varepsilon}} e^{u}} \psi
$$

We multiply this equation by $\psi$, integrate in $\Omega_{\varepsilon}$ and use Proposition 2.2 to obtain

$$
\int_{\Omega_{\varepsilon}}|\nabla \psi|^{2}=\lambda \int_{\Omega_{\varepsilon}} \frac{e^{u}}{\int_{\Omega_{\varepsilon}} e^{u}} \psi^{2} \leq\left(\frac{8}{R_{\varepsilon}^{2}}-\frac{\lambda}{\left|\Omega_{\varepsilon}\right|}\right) \frac{\lambda}{8 \pi-\lambda} \int_{\Omega_{\varepsilon}} \psi^{2} .
$$


By Proposition 3.1, the left hand side of (17) is strictly greater than $4 \pi^{2} \int_{\Omega_{\varepsilon}} \psi^{2}$. Therefore, we deduce

$$
4 \pi^{2}<\left(\frac{8}{R_{\varepsilon}^{2}}-\frac{\lambda}{\left|\Omega_{\varepsilon}\right|}\right) \frac{\lambda}{8 \pi-\lambda}
$$

or equivalently,

$$
\frac{\lambda^{2}}{\left|\Omega_{\varepsilon}\right|}-\left(4 \pi^{2}+\frac{8}{R_{\varepsilon}^{2}}\right) \lambda+8 \cdot 4 \pi^{3}<0 .
$$

Using (13), one sees that the above polynomial is nonpositive at $\lambda=8 \pi$. Therefore, we must have $\lambda>\lambda^{*}(\varepsilon)$, where $\lambda^{*}(\varepsilon) \in(0,8 \pi]$ is the first root of the polynomial. Computing the first root explicitly, we arrive at expression (4) for $\lambda^{*}(\varepsilon)$.

Proof of Corollary 1.2. We recall that Ricciardi and Tarantello [RT98] have established that there exists a nonzero one-dimensional solution of (1) if and only if $\lambda>4 \pi^{2} \varepsilon$.

The first statement of Corollary 1.2 follows from this fact and Theorem 1.1.

To establish the second statement, let $\varepsilon \leq \varepsilon_{0}$. If $\lambda \leq 4 \pi^{2} \varepsilon$ then 0 is the unique solution by part (i), and as a consequence it is also the unique minimizer. Now, if $4 \pi^{2} \varepsilon<\lambda \leq 8 \pi$ then $\inf _{E} \mathcal{J}^{\lambda, \varepsilon}<0$ (see Proposition 4.1(iii) for a proof of this fact, already established in [RT98]). Hence, there exists a nonzero minimizer (and therefore a nonzero solution).

\section{Some properties of $\mathcal{J}^{\lambda, \varepsilon}$ and of its minimiz- ers}

The following result shows that the energy of a minimizer can be estimated from above using the first Bessel function. As a consequence, one obtains that inf $\mathcal{J}^{\lambda, \varepsilon}<0$ for $\lambda>4 \pi^{2} \varepsilon$ (see statement (iii) below). This fact, already established in [RT98], has been used in the proof of Corollary 1.2.

Proposition 4.1. (i) The following equality holds:

$$
I_{0}(t):=\int_{-1 / 2}^{1 / 2} e^{t \cos (2 \pi y)} d y=\sum_{n=0}^{\infty}\left(\frac{t^{n}}{2^{n} n !}\right)^{2} \quad \text { for all } t \in \mathbb{R}
$$

(ii) Let $\alpha \geq 0$ and consider $\Phi_{\alpha}(t):=\alpha t^{2}-\log I_{0}(t)$. Then,

$$
\inf _{t>0} \Phi_{\alpha}(t)<0 \quad \text { if and only if } \quad \alpha<\frac{1}{4} .
$$


(iii) Let $v(x, y)=v(x)=\cos (2 \pi \varepsilon x)$. Then,

$$
\mathcal{J}^{\lambda, \varepsilon}(t v)=\lambda \Phi_{\pi^{2} \varepsilon \lambda^{-1}}(t)
$$

for every $t \in \mathbb{R}$. In particular, if $\lambda>4 \pi^{2} \varepsilon$ then $\mathcal{J}^{\lambda, \varepsilon}(t v)<0$ for some $t>0$.

Proof. (i) Setting $\psi(y):=\cos (2 \pi y)$, we have

$$
\int_{-1 / 2}^{1 / 2} \psi(y)^{2 n+1} d y=0 \quad \text { and } \quad \int_{-1 / 2}^{1 / 2} \psi(y)^{2 n} d y=\frac{(2 n-1)(2 n-3) \cdots 1}{2^{n} n !}
$$

for all integer $n \geq 0$. The last equality follows easily integrating by parts $\psi(y)^{2 n}=\psi(y)^{2 n-1} \cos (2 \pi y)$. Thus,

$$
\begin{aligned}
\int_{-1 / 2}^{1 / 2} e^{t \psi(y)} d y & =\sum_{n=0}^{\infty} \int_{-1 / 2}^{1 / 2} \frac{(t \psi(y))^{2 n}}{(2 n) !} d y=\sum_{n=0}^{\infty} \frac{t^{2 n}}{(2 n) !} \frac{(2 n-1)(2 n-3) \cdots 1}{2^{n} n !} \\
& =\sum_{n=0}^{\infty} \frac{t^{2 n}}{2^{n} n !} \frac{1}{2^{n} n !}=\sum_{n=0}^{\infty}\left(\frac{t^{n}}{2^{n} n !}\right)^{2} .
\end{aligned}
$$

(ii) Using part (i) we have the following equivalences:

$$
\begin{aligned}
\Phi_{\alpha}(t) \geq 0 & \Longleftrightarrow e^{\alpha t^{2}} \geq I_{0}(t) \Longleftrightarrow \sum_{n=0}^{\infty} \frac{\left(\alpha t^{2}\right)^{n}}{n !} \geq \sum_{n=0}^{\infty}\left(\frac{t^{n}}{2^{n} n !}\right)^{2} \\
& \Longleftrightarrow \sum_{n=1}^{\infty}\left(\alpha^{n}-\frac{1}{2^{2 n} n !}\right) \frac{t^{2 n}}{n !} \geq 0 .
\end{aligned}
$$

Now, if $\alpha \geq 1 / 4$ then all coefficients of the series in (18) are nonnegative, and hence $\Phi_{\alpha}(t) \geq 0$ for all $t \geq 0$. On the other hand, if $\alpha<1 / 4$ then the coefficient corresponding to $n=1$ in (18) is negative, and therefore $\Phi_{\alpha}(t)<0$ for $t>0$ small.

(iii) It is easy to check that

$$
\mathcal{J}^{\lambda, \varepsilon}(t \cos (2 \pi \varepsilon x))=\lambda\left(\frac{\pi^{2} \varepsilon t^{2}}{\lambda}-\log I_{0}(t)\right)=\lambda \Phi_{\pi^{2} \varepsilon \lambda^{-1}}(t) .
$$

The second assertion is an immediate consequence of this equality and of statement (ii).

The following result due to Kawohl (see [K85], page 82) establishes that minimizers are Steiner symmetric with respect to both axis, up to a translation. This is an interesting symmetry result that, however, we do not use in this paper. More precisely, we have: 
Proposition 4.2. (Kawohl) Let $u$ be a nonzero minimizer of $\mathcal{J}^{\lambda, \varepsilon}$ with its maximum located at the origin. Then, the following hold:

(i) $u$ is an even function of $x$ and of $y$.

(ii) For every $y$ fixed, $u(x, y)$ is increasing for $x \in(-1 /(2 \varepsilon), 0)$ and decreasing for $x \in(0,1 /(2 \varepsilon))$.

(iii) For every $x$ fixed, $u(x, y)$ is increasing for $y \in(-1 / 2,0)$ and decreasing for $y \in(0,1 / 2)$.

(iv) $\frac{\partial u}{\partial \nu} \equiv 0$ on $\partial \Omega_{\varepsilon}$.

Note that (iv) follows from (ii) and (iii), together with the periodicity of $u$.

\section{A Maximum conformal radius of a rectangle}

We start this appendix recalling the definition and some properties of the conformal radius of a domain.

Let $\Omega$ be a simply connected domain of $\mathbb{R}^{2}$ and let $B_{1}=B_{1}(0)$. Given $q \in \Omega$, there exists a unique conformal map $\varphi_{q}: B_{1} \rightarrow \Omega$, one to one and onto, such that $\varphi_{q}(0)=q$ and $\varphi_{q}^{\prime}(0)>0$. Define the conformal radius of $\Omega$ with respect to $q$ as $R_{\Omega}(q)=\varphi_{q}^{\prime}(0)$, and the maximum conformal radius of $\Omega$ as $R_{\Omega}=\sup _{q \in \Omega} R_{\Omega}(q)$.

It is easy to prove that if $G(p, q)=-\frac{1}{2 \pi} \log |p-q|+w(p, q)$ is the Green's function of $\Omega$ subject to zero Dirichlet boundary conditions, where $w$ is its regular part, then

$$
R_{\Omega}(q)=\exp \{2 \pi w(q, q)\}
$$

If $\Omega \subset \Omega^{\prime}$ and $\Omega \neq \Omega^{\prime}$, then $R_{\Omega}<R_{\Omega^{\prime}}$. Indeed, let $q \in \Omega, \varphi_{q}$ be the unique conformal map defining $R_{\Omega}(q)$, and $\psi_{q}$ be the unique conformal map defining $R_{\Omega^{\prime}}(q)$. Consider the analytic function $\varphi_{q}: B_{1} \rightarrow \Omega^{\prime}$, which is not onto. It follows, by Schwarz Lemma applied to $\psi_{q}^{-1} \circ \varphi_{q}$, that $R_{\Omega}(q)=\varphi_{q}^{\prime}(0)<$ $\psi_{q}^{\prime}(0)=R_{\Omega^{\prime}}(q)$.

In particular, the maximum conformal radius of the rectangle $\Omega_{\varepsilon}$, that we denote by $R_{\varepsilon}$,

$$
R_{\varepsilon}:=R_{\Omega_{\varepsilon}}=R_{(-1 /(2 \varepsilon), 1 /(2 \varepsilon)) \times(-1 / 2,1 / 2)},
$$

is decreasing in $\varepsilon$.

The maximum conformal radius of $\Omega_{\varepsilon}$ is attained at the center, that is, $R_{\varepsilon}=R_{\varepsilon}(0)$. Indeed, if $R_{\varepsilon}$ is attained in $p \in \Omega_{\varepsilon}$ then, as a consequence of the symmetry of $\Omega_{\varepsilon}$, it is also attained in $-p$. That is, $R_{\varepsilon}=R_{\varepsilon}( \pm p)$. Let $\varphi_{p}$ and $\varphi_{-p}$ be the unique conformal maps defining $R_{\varepsilon}(p)$ and $R_{\varepsilon}(-p)$, respectively. Consider $\psi_{p}=\left(\varphi_{p}+\varphi_{-p}\right) / 2: B_{1} \rightarrow \Omega_{\varepsilon}$ (which perhaps is not onto) and note that $\psi_{p}(0)=0$ and $\psi_{p}^{\prime}(0)>0$. Using Schwarz Lemma as before, we 
deduce that $R_{\varepsilon}(0) \geq \psi_{p}^{\prime}(0)=\left(\varphi_{p}^{\prime}(0)+\varphi_{-p}^{\prime}(0)\right) / 2=R_{\varepsilon}$. Hence, the maximum conformal radius is attained at 0 .

An explicit formula for the maximum conformal radius of a rectangle of sides $1 / \varepsilon$ and 1 is given by

$$
R_{\varepsilon}=\frac{2}{\pi}\left(1+2 \sum_{n=1}^{\infty} e^{\frac{-\pi n^{2}}{\varepsilon}}\right)^{-2},
$$

as stated in page 253 of [PS51]. The rest of this section is dedicated to give a proof of it -that we could not find in the literature.

As a consequence of (19), $\lim _{\varepsilon \downarrow 0} R_{\varepsilon}=2 / \pi$ (note that this value can also be computed using a conformal map from the unit disk to the infinite band of width 1 ) and $R_{1} \simeq 0.539$.

To prove (19), we introduce some notation. Consider $\omega_{1}, \omega_{3} \in \mathbb{C}, \omega_{2}:=$ $\omega_{1}+\omega_{3}$ and the lattice $\mathcal{L}:=\mathbb{Z}\left(2 \omega_{1}\right)+\mathbb{Z}\left(2 \omega_{3}\right)$. For our purposes, we always take $\omega_{1}, i \omega_{3} \in \mathbb{R}$.

Definition A.1. Given the lattice $\mathcal{L}$, the $\mathcal{P}$-Weierstrass function is defined by

$$
\mathcal{P}(z):=\frac{1}{z^{2}}+\sum_{w \in \mathcal{L}}\left\{\frac{1}{(z-w)^{2}}-\frac{1}{w^{2}}\right\} .
$$

Set $e_{i}:=\mathcal{P}\left(\omega_{i}\right), i=1,2,3$. Given $\tau \in \mathbb{C}$ and setting $q:=e^{i \pi \tau}$, the third theta function is defined by

$$
\theta_{3}(z \mid \tau)=1+2 \sum_{n=1}^{\infty} q^{n^{2}} \cos (2 n z) .
$$

We define $\theta_{3}(z):=\theta_{3}(z \mid \tau)$ where $\tau$ is chosen by $\tau:=\omega_{3} / \omega_{1}$.

The following properties of these functions can be found in pages 150-163 of [L89]:

1) The function $\mathcal{P}: \mathbb{C} \rightarrow \mathbb{C}$ is doubly periodic, with periods $2 \omega_{1}$ and $2 \omega_{3}$. Moreover, $\mathcal{P}(-z)=\mathcal{P}(z)$.

2) We have $e_{i} \in \mathbb{R}$ and

$$
e_{3}<e_{2}<e_{1}
$$

3) $\mathcal{P}$ restricted to the rectangle $R$ of vertices $O \omega_{1} \omega_{2} \omega_{3}$ maps $R$ onto the upper half-plane.

4) From relation (6.8.12) of [L89] and since $\mathcal{P}$ is even, we deduce

$$
\mathcal{P}\left(\frac{\omega_{2}}{2}\right)=e_{2}+\left\{e_{2}-e_{1}\right\}^{1 / 2}\left\{e_{2}-e_{3}\right\}^{1 / 2} .
$$


5) We have

$$
\mathcal{P}^{\prime}(z)=-2\left\{\mathcal{P}(z)-e_{1}\right\}^{1 / 2}\left\{\mathcal{P}(z)-e_{2}\right\}^{1 / 2}\left\{\mathcal{P}(z)-e_{3}\right\}^{1 / 2},
$$

and

$$
\left(e_{1}-e_{3}\right)^{1 / 2}=\frac{\pi}{2 \omega_{1}} \theta_{3}^{2}(0)
$$

We compose the $\mathcal{P}$-Weierstrass function with a Möbius map to obtain a conformal mapping from the rectangle $R$ to a ball. As a consequence, we obtain the following formula for the maximum conformal radius of $R$.

Proposition A.2. The maximum conformal radius $\dot{R}$ of the rectangle $R$ is given by

$$
\dot{R}=2 \frac{\left|\operatorname{Im}\left\{\mathcal{P}\left(\frac{\omega_{2}}{2}\right)\right\}\right|}{\left|\mathcal{P}^{\prime}\left(\frac{\omega_{2}}{2}\right)\right|}=\frac{2 \omega_{1}}{\pi} \frac{1}{\theta_{3}^{2}(0)} .
$$

Formula (19) follows immediately from (25). Simply take $\omega_{1}=1, \omega_{3}=i / \varepsilon$, and use (20).

Proof of Proposition A.2. Given a conformal map $f$ from $R$ onto the ball $B_{1}(0)$, mapping $\omega_{2} / 2$ to 0 , the maximum conformal radius of $R$ is given by $\left|f^{\prime}\left(\omega_{2} / 2\right)\right|^{-1}$.

On the one hand, the Weierstrass function maps conformally $R$ onto the upper half-plane. On the other hand, by setting $p_{0}:=\mathcal{P}\left(\omega_{2} / 2\right)$, the Möbius transformation

$$
h: z \mapsto \frac{z-p_{0}}{z-\bar{p}_{0}}
$$

maps the upper half-plane onto $B_{1}(0)$. Therefore, $h \circ \mathcal{P}$ is a conformal map from $R$ onto $B_{1}(0)$ mapping $\omega_{2} / 2$ to 0 . Now,

$$
(h \circ \mathcal{P})^{\prime}\left(\frac{\omega_{2}}{2}\right)=\frac{\mathcal{P}^{\prime}\left(\frac{\omega_{2}}{2}\right)}{\mathcal{P}\left(\frac{\omega_{2}}{2}\right)-\overline{\mathcal{P}\left(\frac{\omega_{2}}{2}\right)}}=\frac{1}{2} \frac{\mathcal{P}^{\prime}\left(\frac{\omega_{2}}{2}\right)}{\operatorname{Im} \mathcal{P}\left(\frac{\omega_{2}}{2}\right)},
$$

which yields the first equality of (25).

Let us now prove the second equality of (25). On the one hand, using (22) and (21), we deduce that

$$
\operatorname{Im}\left\{\mathcal{P}\left(\frac{\omega_{2}}{2}\right)\right\}=\left(e_{1}-e_{2}\right)^{1 / 2}\left(e_{2}-e_{3}\right)^{1 / 2} .
$$

On the other hand, by (23) and (22) we obtain

$$
\begin{aligned}
\left\{\mathcal{P}^{\prime}\left(\frac{\omega_{2}}{2}\right)\right\}^{2} & =4\left\{\mathcal{P}\left(\frac{\omega_{2}}{2}\right)-e_{1}\right\}\left\{\mathcal{P}\left(\frac{\omega_{2}}{2}\right)-e_{2}\right\}\left\{\mathcal{P}\left(\frac{\omega_{2}}{2}\right)-e_{3}\right\} \\
& =4\left(e_{2}-e_{1}\right)\left(e_{2}-e_{3}\right)\left\{\left(e_{2}-e_{1}\right)^{1 / 2}+\left(e_{2}-e_{3}\right)^{1 / 2}\right\}^{2}
\end{aligned}
$$


Therefore,

$$
\begin{aligned}
\left|\mathcal{P}^{\prime}\left(\frac{\omega_{2}}{2}\right)\right|^{2} & =4\left|e_{2}-e_{1}\right|\left|e_{2}-e_{3}\right|\left|\left(e_{2}-e_{1}\right)^{1 / 2}+\left(e_{2}-e_{3}\right)^{1 / 2}\right|^{2} \\
& =4\left|e_{2}-e_{1}\right|\left|e_{2}-e_{3}\right|\left|i\left(e_{1}-e_{2}\right)^{1 / 2}+\left(e_{2}-e_{3}\right)^{1 / 2}\right|^{2} \\
& =4\left|e_{2}-e_{1}\right|\left|e_{2}-e_{3}\right|\left|\left(e_{2}-e_{3}\right)+\left(e_{1}-e_{2}\right)\right| \\
& =4\left|e_{2}-e_{1}\right|\left|e_{2}-e_{3}\right|\left|e_{1}-e_{3}\right| .
\end{aligned}
$$

From (26), (27) and (24), we finally get

$$
2 \frac{\left|\operatorname{Im}\left\{\mathcal{P}\left(\frac{\omega_{2}}{2}\right)\right\}\right|}{\left|\mathcal{P}^{\prime}\left(\frac{\omega_{2}}{2}\right)\right|}=\frac{1}{\left|e_{1}-e_{3}\right|^{1 / 2}}=\frac{2 \omega_{1}}{\pi} \frac{1}{\theta_{3}^{2}(0)},
$$

which is the second equality of (25).

\section{Acknowledgements}

The first and the third authors were supported by MCYT grant BMF200204613-C03. The first author was also supported by MCYT grant BFM20000962-C02-02, and by a Harrington Faculty Fellowship at The University of Texas at Austin.

The second author was supported by TMR network FMRX-CT98-0201, NSFC and Swiss National Foundation Contract No. 8220-64676. Part of this work was done while the second author visited the Universitat Politècnica de Catalunya (UPC) and Rutgers University. He thanks the Departament de Matemàtica Aplicada I of the UPC and the Department of Mathematics in Rutgers for their great hospitality.

We are greatful to Yanyan Li and M. Kiessling for useful discussions and to B. Kawohl for bringing Proposition 4.2 to our attention.

\section{References}

[B73] C. Bandle, Mean value theorems for functions satisfying the inequality $\Delta u+K e^{u} \geq 0$, Arch. Rational Mech. Anal. 51 (1973), 70-84.

[B80] C. Bandle, Isoperimetric inequalities and applications, Pitman, London, 1980.

[CLMP92] E. Caglioti, P.L. Lions, C. Marchioro, M. Pulvirenti, A special class of stationary flows for two-dimensional Euler equations: a statistical mechanics description, Comm. Math. Phys. 143 (1992), 501525 . 
[CL] C.C Chen, C.S. Lin, Sharp estimates for solutions of multi-bubbles in compact Riemann surfaces, Comm. Pure Appl. Math. 55 (2002), 728-771.

[DJLW97] W. Ding, J. Jost, J. Li, G. Wang, The differential equation $\Delta u=$ $8 \pi-8 \pi h e^{u}$ on a compact Riemann surface, Asian J. Math. 1 (1997), $230-248$.

[DJLW98] W. Ding, J. Jost, J. Li, G. Wang, An analysis of the two-vortex case in the Chern-Simons Higgs model, Calc. Var. 7 (1998), 87-97.

[D96] G. Dunne, Self-dual Chern Simons theories, Lecture Notes in Physics, New Series M 36, Springer, New York, 1996.

[H64] P. Hartman, Geodesic parallel coordinates in the large, Amer. J. Math. 86 (1964), 705-727.

[HKP90] J. Hong, Y. Kim, P.Y. Pac, Multivortex solutions of the abelian Chern Simons theory, Phys. Rev. Lett. 64 (1990), 2230-2233.

[JW90] R. Jackiw, E.J. Weinberg, Selfdual Chern Simons vortices, Phys. Rev. Lett. 64 (1990), 2234-2237.

[K85] B. Kawohl, Rearrangements and convexity of level sets in PDE, Lecture Notes in Mathematics 1150, Springer-Verlag, 1985.

[Ki93] M. K.-H. Kiessling, Statistical mechanics of classical particles with logarithmic interactions, Comm. Pure Appl. Math. 47 (1993), 27-56.

[Ki95] M. K.-H. Kiessling, Negative temperature bounds for $2 D$ vorticity compounds, Lett. Math. Phys. 34 (1995), 49-57.

[L89] D.F. Lawden, Elliptic functions and applications, Appl. Math. Sci. 80, Springer-Verlag, Berlin, 1989.

[M71] J. Moser, A Sharp Form of an Inequality by N. Trudinger, Indiana U. Math. J. 20 (1996), 1077-1092.

[NT98] M. Nolasco, G. Tarantello, On a sharp Sobolev type inequality on two dimensional compact manifolds, Arch. Rational Mech. Anal. 145 (1998), 161-195.

[NT99] M. Nolasco, G. Tarantello, Double vortex condensates in the ChernSimons-Higgs theory, Calc. Var. Partial Diff. Eq. 9 (1999), 31-94. 
[PS51] G. Pólya, D. Szegö, Isoperimetric inequalities in mathematical physics, Princeton University Press, Princeton, 1951.

[RT98] T. Ricciardi, G. Tarantello, On a periodic boundary value problem with exponential nonlinearities, Diff. Int. Eq. 11 (1998), 745-753.

[ST98] M. Struwe, G. Tarantello, On multivortex solutions in Chern-Simons Gauge theory, Boll. U.M.I. B 1 (1998), 109-121.

[S92] T. Suzuki, Global analysis for a two-dimensional elliptic eigenvalue problem with the exponential nonlinearity, Ann. Inst. H. Poincaré, Anal. Non Linéaire 9 (1992), 367-397.

[S94] T. Suzuki, Semilinear elliptic equations, GAKUTO International Series. Mathematical Sciences and Applications 3, Tokyo, 1994.

[T96] G. Tarantello, Multiple condensate solutions for the Chern-SimonsHiggs theory, J. Math. Phys. 37 (1996), 3769-3796. 